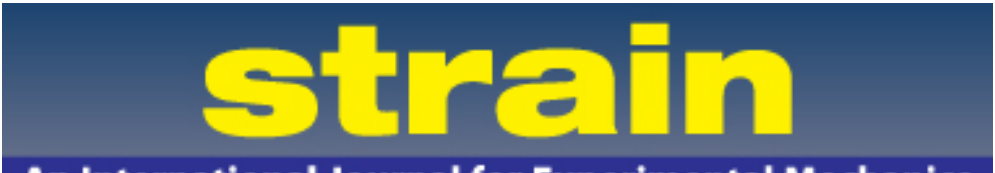

An International Journal for Experimental Mechanics

\title{
Procedure for accurate calculation of the J-integral from Digital Volume Correlation displacement data
}

\begin{tabular}{|r|l|}
\hline Journal: & Strain \\
\hline Manuscript ID & Draft \\
\hline Manuscript Type: & Full Paper \\
\hline Author: & n/a \\
\hline Complete List of Authors: & $\begin{array}{l}\text { Becker, Thorsten; University of Stellenbosch, Mechanical and } \\
\text { Mechatronic Engineering } \\
\text { Molteno, Matthew; University of Stellenbosch, Mechanical and } \\
\text { Mechatronic Engineering } \\
\text { Marrow, James; University of Oxford, Materials }\end{array}$ \\
\hline Keywords: & $\begin{array}{l}\text { digital volume correlation, fracture mechanics, J-integral, X-ray } \\
\text { computed tomography }\end{array}$ \\
\hline &
\end{tabular}

\section{SCHOLARONE \\ Manuscripts}




\title{
Procedure for accurate calculation of the $J$-integral from Digital Volume Correlation displacement data
}

\author{
Becker T.H. ${ }^{*}$, Molteno M.R. ${ }^{1}$, Marrow T.J. ${ }^{2}$ \\ ${ }^{1}$ Department of Mechanical and Mechatronic Engineering, University of Stellenbosch, \\ Joubert Street, Stellenbosch, 7600, RSA \\ Tel. +27(0)21 808 4045, Email. tbecker@sun.ac.za \\ 2 Department of Materials, University of Oxford, Oxford OX1 3PH, UK
}

\begin{abstract}
The application of Digital Image and Volume Correlation techniques to obtain displacement fields from images has become ubiquitous in experimental fracture mechanics. In this paper, a procedure to extract the J-integral $(J)$ from three-dimensional displacement fields obtained using digital volume correlation is presented. The procedure has been specifically adapted to allow for experimental noise and errors, such as poorly defined crack front displacements, smearing of the displacement field across the crack faces and knowledge of the imprecise crack front location. The implementation is verified using analytical crack tip fields perturbed with synthetic image correlation errors to characterise the response of $J$. The method is then applied to experimental results using a Magnesium alloy WE43 loaded elastically in mixed-mode. The steps outlined are intended as a guideline for application of the volume integral from displacement fields to allow for accurate calculation of $J$ along a crack front embedded within the volume.
\end{abstract}

\section{Keywords}

digital volume correlation, fracture mechanics, J-integral, X-ray computed tomography 


\section{Symbols}

$A_{c}, \hat{A}_{c} \quad$ Crack extension in the global and reference state respectively

$\sigma_{i j} \quad$ Cauchy stress tensor

$\varepsilon_{i j} \quad$ Infinitesimal strain tensor

$U_{i}, u_{i}, \hat{u}_{i}$ Displacement vector in the global, interpolated and reference state respectively

$w \quad$ Strain energy density

$V, \bar{V} \quad$ Integration volume in the global and reference state respectively

$q \quad$ Crack extension function

$v \quad$ Poisson's ratio

E Young's modulus

J J-integral

$S \quad$ Crack front

$n \quad$ Crack front segment

$\Delta S \quad$ Integration volume breadth $\left(x_{3}\right)$

$x_{i}, \hat{x}_{i} \quad$ Co-ordinate system in the global and reference state respectively

$\Delta x \quad$ Integration volume width $\left(x_{1}\right)$ or height $\left(x_{2}\right)$

$O_{i} \quad$ Crack tip location

$L_{i} \quad$ Crack front direction

$M_{i} \quad$ Crack normal direction

$N_{i} \quad$ Crack propagation direction

$P_{i}^{1}, P_{i}^{2} \quad$ Start and end points of crack path.

$\boldsymbol{R} \quad$ Rotation matrix

$\hat{r}_{m} \quad$ mask height in reference state

$\boldsymbol{t}$ displacement vector

$\boldsymbol{T}$ Transformation matrix 


\section{Introduction}

The application of Digital Image Correlation (DIC) and Digital Volume Correlation (DVC) techniques to obtain displacement data fields from images has become ubiquitous in experimental fracture mechanics. Many examples now exist that demonstrate the successful extraction of fracture parameters, such as stress-intensity factors obtained by McNeill et al. [1], Lim et al. [2] and Roux et al. [3], mixed-mode problems addressed by Rethore and Gravouil [4], Yoneyama et al. [5,6] and Sistaninia et al. [7] and energy approaches such as the J-integral by Yoneyama et al. [8] and the authors of this work $[9,10]$, to name but a few.

A significant limitation of image correlation approaches (compared to others such as Moire interferometry) is the reduced accuracy experienced near the strain concentration at the crack tip. State-of-the art subset based correlation techniques suffer from smearing of information across the crack faces resulting in spurious displacement and strain readings. It is therefore imperative for DIC and DVC based fracture property extraction methods to account for these sources of error by: i) using significantly more complex (and generally in-house developed) correlation techniques (e.g. subset splitting [11]); ii) replacing poorly defined or missing data in these regions (e.g. with numerical approximations of the material after the crack front has been found $[12,13])$; or iii) developing methods that allow for the selection of data remote from the crack tip thereby eliminating the need for accurate displacement data near the crack faces and crack front [10].

Similarly, the knowledge of the crack tip location may be critical when extracting fracture parameters from experimentally obtained displacement fields. Often, it is not possible to define an exact crack tip or front location and complex segmentation and optimisation techniques have been developed that aim to best identify the crack tip location $[14,15]$.

Random permutations in the signal (light or x-ray) also result in noise in the individual vectors of the displacement fields. DIC noise is typically reported at 0.01 and 0.02 pixel for in-plane and out-of-plane respectively [16], while DVC tends to achieve much lower accuracies, which are typically reported as 0.05 to 0.1 voxels [17]. This is mainly due to the reliance of DVC on there being a suitable distribution (shape, size, brightness) of natural features within the material volume [18], whereas DIC can utilise artificially applied and optimal speckle patterns with high contrast.

Some of these problems can be overcome when using field fitting approaches, whereby optimal analytical solutions to crack tip displacement fields are fitted to the measured displacement data [5]. However, field fitting approaches may be ill-suited to cases where the crack tip region is poorly defined, as in large-scale plasticity, crack branching, or micro-cracking (e.g. graphite or concrete) [19]. Furthermore, displacement fields in regions near the crack tip are a challenge to measure [20]. This is because both DIC and DVC usually assume a small set of deformation parameters (i.e. translation, dilatation and shear strains and in some cases rotation) for efficiency purposes. As a result, sharp discontinuities and crack tip singularities tend to be inconsistent with the assumed deformations within a subset (region of analysis), causing poor correlation in crack tip regions [11,21]. As such, the J-integral provides better suited tool for extracting, from experimentally determined displacement fields, the fracture parameters that describe the action on the crack tip. In the DIC form, as previously presented by the authors $[9,10]$, high strain regions near the crack can be omitted under the assumption of a plane stress state, provided that the crack path does not leave the J-integral contour. In the volumetric form of the J-integral [22], the displacement fields around the crack front are required since the 
integral encapsulates a predefined region of interest that necessitates data along the crack front [22].

This presents a problem when using DVC computed displacement fields to obtain J-integral based fracture parameters. The poorly defined displacement fields, in combination with the need of accurate displacement data around the crack front, creates a need for an unique implementation of the J-integral. One method to overcome this is the replace the missing, or censored, data using the Finite Element (FE) framework, as presented by Bahrli et al. [12]. However, this requires extrapolation from the measured data in order to define a displacement field in the cracked region, which in turn requires assumptions on the material properties (plasticity, microcracking) in this region, and the method cannot consider the effects of complex fracture mechanics such as crack branching or bridging. This extrapolation also requires knowledge of the precise crack front location. Another method, which the authors are aware of, is to adapt the volumetric formulation to allow for poorly defined crack front displacement data. However, such work has not been reported in the open literature.

We present an alternative procedure for computing the J-integral directly from DVC displacement data. The work is therefore similar to the analysis performed on surface $\mathrm{J}$ integrals that has previously been presented by the authors $[9,10]$. This paper presents a procedure and formulation of the J-integral that is better suited for DVC displacement data. Typical sources of errors are studied using synthetically generated displacement fields and errors. Subsequently, the procedure is applied to an experimental case study. The experimental results are obtained at sub-critical loads, with elastic deformation, and validation is performed against simulations using the finite element software Abaqus that uses the volume integral as detailed in $[22,23]$.

\section{Three-dimensional framework}

In this work, the premise for the three-dimensional (volumetric) framework of the J-integral $(J)$ is that global DVC computed displacement data is available in the vicinity of the crack front in a Cartesian co-ordinate system $\boldsymbol{X}_{i}$ given by $U_{i}$, and that the mechanical (deformation) properties for the material are known (i.e. the relationship between the strain and stress tensors). To subsequently compute $J$ values along the crack front, the volumetric form presented by Shih et al. [22] is adopted. It is noted that the volumetric $J$ formulation differs from the classical two-dimensional formulation in that the contour is not represented by a line or area, but as a surface encapsulating a specified region, i.e. volume, $V$.

$J$ is calculated for $V$, which is centred at $O_{i}$ along the crack front $S_{i}$ as show in Error! Reference source not found.. A convention is used where the global coordinates system $\boldsymbol{X}_{i}$ is designated by an uppercase and a local Cartesian co-ordinate system $\boldsymbol{x}_{i}$ is designated by a lowercase at the crack front. The local co-ordinate system is centred at $O_{i}$ with $\boldsymbol{x}_{1}$ in the crack plane and perpendicular to the crack front, $\boldsymbol{x}_{2}$ normal to the crack plane and $\boldsymbol{x}_{3}$ tangential to the crack front.

Similar to the adaptation of the line integral to an equivalent domain integral in the twodimensional formulation, the volumetric formulation takes on an equivalent volumetric domain integral given by Eq. (1). The volumetric integral allows for more data points to be considered, compared to alternative formulations, and is therefore better suited for full-field data [9]. 


$$
J=\frac{1}{A_{c}} \int_{V}\left(\sigma_{i j} \frac{\partial u_{i}}{\partial x_{1}}-w \delta_{1 j}\right) \frac{\partial q}{\partial x_{j}} d V
$$

Eq. (1) contains a displacement derivative, $\partial u_{i} / \partial x_{1}$, in the $\boldsymbol{x}_{1}$ direction, a Cauchy stress tensor, $\sigma_{i j}$, a strain energy density, $w$ and $\partial q / \partial x_{j}$, which is the derivative of a virtual crack extension function, $q$, defined by Eq. (5). $A_{c}$ is obtained using Eq. (6) and is directly dependant on the choice in $q . \delta_{1 j}$ is the Kronecker delta.

$\sigma_{i j}$ is calculated using Eqs. 2 and 3. In this work, Eq. (2) is given under the assumptions of a linear-elastic isotropic material, however, more complex mechanical behaviour models may be used such as presented by [12].

$$
\sigma_{i j}=\frac{E}{1+v}\left[\varepsilon_{i j}+\frac{v}{1-2 v} \varepsilon_{k k} \delta_{i j}\right]
$$

$E$ and $v$ are the Young's modulus and Poisson's ratio respectively. $\varepsilon_{i j}$ is the symmetric strain tensor calculated using the displacement gradients, $\partial u_{i} / \partial x_{j}$, in Eq. (3).

$$
\varepsilon_{i j}=\frac{1}{2}\left(\frac{\partial u_{i}}{\partial x_{j}}+\frac{\partial u_{j}}{\partial x_{i}}\right)
$$

The strain energy density, $w$, is calculated using Eq. (4).

$$
w=\int \sigma_{i j} d \varepsilon_{i j}
$$

The crack extension function, $q$, requires a function that is sufficiently smooth and that is constrained at its boundaries by Eq. (5)

$$
q=\left\{\begin{array}{lc}
1 & \text { on } O_{i} \\
0 & \text { on the outer edge of } V
\end{array}\right.
$$

$A_{c}$ is obtained by integrating $q$ along crack front segment $\Delta S$ as defined by Shih et al. [22] using Eq. (6).

$$
A_{c}=\int_{\Delta s} q d S
$$

Eqs. 2 and 3 assume material specific mechanical behaviour properties within $V$ and so $J$ is computed on the basis that the mechanical (deformation) behaviour is known. In the presented form, $J$ is a function of $U_{i}, E$ and $v$. No load or crack length measurements are required to evaluate $J$.

The distinct feature of the $J$-integral is its contour independence. In the two-dimensional formulation, with an absence of out-of-plane $\left(\boldsymbol{x}_{3}\right)$ stresses under a plane stress assumption (or strains, under a plane strain assumption), contours can be arbitrarily chosen so that the crack tip region is excluded. This significantly reduces error in the computation of $J$ and is especially 
important when considering experimental data that have a significantly higher error than, for example, finite element computed data [9]. In the three-dimensional formulation, the contour will inherently include crack tip data as out-of-place stresses are present [22]. Therefore, the contours cannot be taken in such a way that the crack tip region is excluded. However, as shown in Section 3, careful consideration of $q$ can allow for a computation of $J$ from experimental data with significantly reduced error without affecting contour independence.

\section{Numerical implementation}

Consider the crack front depicted in Error! Reference source not found.a. In the presented approach, three aspects are required for the computation of $J$ from the respective DVC displacement data, namely crack-tip segmentation, integration volume mapping, masking and summation of the integrand. The procedure is outlined below and the implementation is done using Matlab ver. 9.6 software package, where the DVC computed displacement field data with the respective position data are imported into Matlab's workspace.

\subsection{Crack tip segmentation}

The first step requires $V$ to be aligned with the crack front and therefore necessitates knowledge of the crack tip location and orientation. Since $J$ is computed at specific locations along the crack front, the number of $J$ values obtained is dependent on the choice in $n$ number of segments. Using the procedure outlined by [24], $S_{i}$ is divided in $n$ segments, between $P_{i}^{1}$ and $P_{i}^{2}$ with each segment located at $O_{i}^{n}$ with known orientation $\boldsymbol{L}^{n}$ (crack path), $\boldsymbol{M}^{n}$ (crack direction) and $\boldsymbol{N}^{n}$ (crack normal) as depicted in Error! Reference source not found.a. Each segment has a width of $\Delta S$. In this work, the crack tip location and direction is visually, however, other methods are available $[12,15,25]$.

\subsection{Integration Volume mapping}

The second step involves mapping $V$ to the respective crack tip location and orientation. For this, additional steps are required that are outlined below, which involve: i) setting up a reference volume $\hat{V}$; ii) mapping of the reference volume in the global domain; iii) extracting nodal displacements by means of interpolation; and iv) mapping these back to the reference state.

i) $\hat{V}$ is of unit size, has an orientation that is aligned with the horizontal, vertical and normal coordinate system for $\hat{\boldsymbol{x}}_{1}, \hat{\boldsymbol{x}}_{2}$ and $\hat{\boldsymbol{x}}_{3}$ respectively, the crack plane is aligned with $\hat{\boldsymbol{x}}_{1}$, the crack tip is located the origin, and the crack is assumed to propagating in the $\hat{\boldsymbol{x}}_{1}$ direction, i.e. the crack normal aligned with $\hat{\boldsymbol{x}}_{2}$ as shown in in Error! Reference source not found.a. A convention is adopted, whereby the reference volume's symbols use a top hat.

The reference volume has regularly spaced position data in $\hat{\boldsymbol{x}}_{i}$ to allow for simple differentiation and integration, as shown in Error! Reference source not found.b (crosssection of the mid-plane). No advantage is seen in numerical differentiation and integration approaches that require point wise evaluation (i.e. such as in FE implementation). This requires the knowledge of shape function derivatives and their respective Jacobian at each data point. The relatively noisy nature of DVC coupled with the inherent subset smearing does not, in the authors' opinion, warrant such implementation complexities. It is shown 
later, that the choice in $q$ has the greatest influence when dealing with smeared and noisy crack tip data and crack tip position accuracy.

The crack extension function, $q$, is defined as per Eq. (5) and is a function of $\hat{\boldsymbol{x}}_{i} . J$ is written with respect to the local co-ordinate system of $\hat{V}$, with $q$ defined as vector components in which $J$ is the projection onto the $q$ vector field $\left(J=J_{i} q_{i}(i=1,2,3)\right)$. The form given in Eq. (5) is equivalent to computing $J$ in vector form if $q_{i}$ is pointing in the crack growth direction (i.e. $q_{2}=q_{3}=0$ ). The reader is referred to Kuna [26] for more details on the vector form of the volume integral.

Three functions are considered for $q$. It is noted that for the evaluation of $J$, Eq. (1) requires the derivates of $q$ with respect to $\hat{\boldsymbol{x}}_{i}$. Thus, the choice in $q$ is such to either allow for simple numerical implementation (i.e. constant gradients) or to inherently remove poorly defined displacement data near the crack tip (i.e. by setting a near zero gradient at the crack tip). A representation of the $q$ function derivatives, $\partial q / \partial \hat{x}_{j}$, is shown in Error! Reference source not found.

The choice of a 'linear' function results in constant gradient with a minimal gradient value. A polynomial (in this work a 4th order polynomial was chosen) results in steeper gradient at the outer edges in $\hat{V}$ (away from the crack tip) and near zero gradient at the crack tip region. A piecewise linear function (termed 'contour') results in a constant gradient near the outer edges in $\hat{v}$ and zero gradients at the crack tip. As mentioned above, the inherent nature of $J$ in the volume, which encapsulates a segment along a crack front, is that crack tip data will always be included. As such the contour function does not consider a piecewise linear function in $\hat{\boldsymbol{x}}_{3}$. This ensures that the gradients in $\hat{\boldsymbol{x}}_{3}$ are at a minimum near the crack tip. $\hat{A}_{c}$ is based on the choice in $q$, and is calculated using Eq. (6).

A mask is defined over $\hat{V}$ that is centred along the crack (Fig. 2b). The mask defines those displacement data points that are judged to have been significantly smeared near the crack and therefore are not considered to be accurate enough for the evaluation of $J$. In the present analysis of a planar crack, the mask is cuboid in shape, has unit thickness, a length of 0.5 and defined by height $\hat{r}_{m}$, as shown in Error! Reference source not found.b. Data points included in the mask are replaced by means of extrapolation (detailed in Section 3.3).

ii) Mapping of $U_{i}$ to $\hat{V}$ is achieved by mapping the position co-ordinates of the reference volume domain $\hat{\boldsymbol{x}}_{\boldsymbol{i}}$ to the global domain $\boldsymbol{x}_{\boldsymbol{i}}$. This operation requires the knowledge of a transformation matrix $\boldsymbol{T}$ at each integral segment computation $n . \boldsymbol{T}^{n}$ comprises of a scaling matrix $\boldsymbol{\alpha}$ (taken as constant across all segments), a rotation matrix $\boldsymbol{R}^{n}$ and a translation vector $\boldsymbol{t}^{n}$, which are assembled as given in Eq. (7).

$$
\boldsymbol{T}^{n}=\left[\begin{array}{cc}
\boldsymbol{\alpha}^{n} \boldsymbol{R}^{n} & 0 \\
\boldsymbol{t}^{n} & 1
\end{array}\right]
$$

$\boldsymbol{\alpha}$ is a diagonal matrix and determines the size of $V$. In this work $\alpha_{11}=\alpha_{22}=c$ so that the volume length and height are defined by $c$ and are equal in $\boldsymbol{x}_{1}$ and $\boldsymbol{x}_{2} . \alpha_{33}=\Delta S$ represents a volume width that is equal to the segment length. $\boldsymbol{R}^{n}$ is dependent on the crack tip orientation and is obtained using Eq. (8). $\boldsymbol{t}^{n}$ is dependent on the crack tip position and is obtained using Eq. (9). 


$$
\boldsymbol{R}^{n}=\left[\boldsymbol{M}^{n}, \boldsymbol{N}^{n}, \boldsymbol{L}^{n}\right]
$$

$$
\boldsymbol{t}^{n}=\boldsymbol{O}^{n}
$$

The mapping of the $\hat{V}$ to $V$ is undertaken by using Eq. (10).

$$
\left[\begin{array}{c}
\boldsymbol{x} \\
1
\end{array}\right]=\boldsymbol{T}^{n}\left[\begin{array}{l}
\hat{\boldsymbol{x}} \\
1
\end{array}\right]
$$

iii)Interpolation of the displacement field is undertaken using linear interpolation to obtain displacement values at $\boldsymbol{x}$. The interpolation method uses a Delaunay triangulation method as outlined by [27]. Similar to the argument presented in i) it is in the authors' opinion that higher order interpolation schemes do not improve the accuracy in the computation of $J$. In fact, it is argued that due to the noise nature of DVC data, higher interpolation schemes may result in an increase of error as a result of Runge's phenomenon [28].

iv) Subsequently, the interpolated displacement field is mapped back to the reference state using Eq. (11).

$$
\hat{u}_{i}=\left[\boldsymbol{R}^{n}\right]^{T} u_{i}
$$

where $\left[\boldsymbol{R}^{n}\right]^{T}$ is the transpose of $\boldsymbol{R}^{n}$ and $u_{i}$ is the interpolated displacement data. $A_{c}$ is scaled by $\Delta S$ to compute its equivalent value in the reference state, i.e. $\hat{A}_{c}=A_{c} \cdot \Delta S . d V$ is scaled by $2 \Delta S \alpha$ to compute its equivalent value in the reference state, i.e. $d \hat{V}=c^{2} \Delta S$.

\subsection{Masked data}

Before the computation of $J$, it is important to address the masked data close to the crack faces, particularly if a mixed-mode type loading case is expected. Similar to the method presented by the author's for computing mixed-mode J-integral solutions from surface displacement data [9], masked field data is replaced using an iterative least-squares polynomial fitting method developed by Garcia et al. [29] and Wang et al. [30]. The number of iterations used is 100. It is noted that this approach is limited to low displacement gradient regions and smaller masked areas. This is due to the inherent inability of polynomial functions to represent crack tip fields that represent discontinuous and singular values.

\subsection{Summation of the integrand}

The numerical integration of $\hat{V}$ requires the computation of its displacement gradients $d \hat{u}_{i} / d \hat{x}_{j}$, which in turn are used to compute the strain tensor using Eq. (4), the stress tensor using Eq. (3) and the strain energy density using Eq. (5) at each data point in $\hat{x}_{i}$. The choice of $q$ determines $d q / d \hat{x}_{i}$ and $\hat{A}_{c}$ as illustrated in Fig. 3. The computation of $J$ then becomes a simple summation of the terms listed in Eq. (1). 


\section{Errors of the volume J-integral}

There several sources of error to consider when computing $J$ from DVC displacement data. Firstly, as with any experimental measurement technique, the DVC computed displacement field contains random noise. Furthermore, regions near the crack faces and tip are prone to errors. These can arise from the effects of image artefacts introduced in the reconstruction process [31], or may be due to DVC smearing at the crack faces [32]. Secondly, knowledge of the crack alignment and location is required in order to position the integration volume, and while sophisticated crack segmentation algorithms exist to aid segmentation, these always include some measure of uncertainty [32]. Thirdly, a reasonably accurate knowledge of material properties is required in order to compute stress values from the strain field [10].

A pragmatic approach was adopted to quantify the influence of the above errors in the computation of $J$. Synthetic displacement data were generated using the finite element (FE) method, whereby a known error was imposed, and its effect in the computation of $J$ was therefore isolated. Furthermore, $J$ 's contour independence served as an indicator to assess valid computations. Contour independence was inferred when fluctuations of less than 5\% over five contours were achieved. Three contour type functions were considered, as shown in Fig. 3; the contour (piecewise linear) function used a contour width of three.

The error analysis utilised a parallel approach of an Abaqus and an in-house developed linearelastic FE model. A unit size cuboid geometry was generated, with the crack tip orientated at the origin (Fig. 4). The Abaqus model (ver. 6.13) comprised 65800 quadratic 20-node brick elements (C3D20) with collapsed quarter points along the crack front. J-integral values were computed over ten contours using Abaqus' built-in functionality. The in-house developed FE model comprised of 127500 linear eight-node brick elements. The grid of element nodes were arranged in such a way that the computation of $\mathrm{J}$ does not require any interpolation (Section 3.2.iii) and thereby errors due to interpolation are excluded (no crack tip mesh refinement was undertaken). $J$ values were computed in this model, using the above-presented technique, over ten contours. Both models used Young's modulus of $10 \mathrm{GPa}$ and a Poisson's ratio of 0.3. Displacement boundary conditions were applied at the top and bottom surface nodes of the unit size cuboid, such that the sample is loaded either in a crack opening mode (i.e. in the $U_{2}$ direction, designated as $B C_{1}$ ), in-plane shear (i.e. in the $U_{1}$ direction, designated as $B C_{2}$ ), outof-plane shear (i.e. in the $U_{3}$ direction, designated as $B C_{3}$ ) or a combination of the three (designated as $\left.B C_{1,2,3}\right)$. Fig. 4a illustrates the application of $B C_{1}$.

The error analysis presented in Fig. 5 and 6 considered a Monte Carlo analysis of 1000 runs applied on the in-house FE generated displacement data, providing the influence on the computation of $J$ of normally distributed random displacement noise and rigid body (crack tip) misalignment. The respective displacement boundary conditions are applied in such a way that an average $J$ along the crack path of unity is obtained. For $B C_{1}, B C_{2}, B C_{3}$ and $B C_{1,2,3}$ this equated to displacement magnitudes of $7.45 \cdot 10^{-2}, 2.96 \cdot 10^{-1}, 2.98 \cdot 10^{-1}$ and $5 \cdot 10^{-2}$ units respectively. The error in $J$ was subsequently calculated on the basis of the difference between the unit value (i.e. no error) and error analysis for each crack segment along the crack path, expressed as a percentage.

It is worth noting, that the above presented error analysis did not consider the influence of reconstruction and crack smearing errors on the computation of $J$. Initial analysis showed that 
these sources of error do not result in contour independence and therefore do not obtain valid $J$ computations. Errors in the knowledge of material properties are simply a scaling error when a linear-elastic material is assumed, and so have not been considered.

Fig. 5 shows the mean, upper and lower bounds of displacement noise versus the error in the computation of $J$. Considering a typical limit of DVC measurement accuracy of approximately 0.1 voxels (LaVision product manual, DaVis 8.4) and the unit size sample geometry, an assumed (typical for experimental data) image volume of $1000 \times 1000 \times 1000$ voxels would equate to a displacement accuracy of $1 \cdot 10^{-4}$ units. For $B C_{1}, B C_{2}$ and $B C_{3}$ this equate to a $1.34 \%, 0.0338 \%$ and $0.0336 \%$ displacement noise respectively. Similarly, Fig. 6 shows the mean, upper and lower bounds of crack tip misalignment (i.e. rigid body movement of $V$ in $X_{1}$ and $X_{2}$ directions) to establish the sensitivity of $J$ with respect to crack tip alignment. The percentage misalignment reported is with respect to the integration volume size, which at its limit (10 contours) represents 200 voxels or 0.2 units (i.e. a $1 \%$ crack tip location error is equivalent to 2 voxels or $2 \cdot 10^{-3}$ units). Since the fracture mechanics framework uniquely represents the displacement field around any crack tip, an equivalent error estimate in $J$ may be calculated by scaling the calculated $J$ by the error estimates shown in Fig. 5 and 6 with the $J$ value obtained in other experiments (for an approximately similar displacement accuracy).

Fig. 5 and 6 illustrate two features: Firstly, a shearing type loading results in a significantly higher error. This is consistent with findings reported for $J$ values computed from DIC (surface) data [9] and is attributed to the higher strain energy density values along the crack faces. It highlights the importance of adequate masking and data replacement when mixed-mode loading is expected. Further testing, not shown, included a mask of varying width $\hat{r}_{m}$. The results show that the data replaced using the iterative least-squares polynomial fitting (Section 3.3) was robust in maintaining contour independence and accurate $J$ computations. Secondly, the type of $q$ function (Fig. 3) has a notable effect on the $J$ error. A compromise exists - a linear $q$ function shows less sensitivity to random displacement noise, whereas a contour type function shows an order of magnitude reduction in error for crack tip location. The polynomial function does not seem to offer any significant improvement for either of these considerations. This compromise exists due to the gradients of the $q$ function. As mentioned above, a linear $q$ results in the lowest possible gradients and thereby, the displacement noise error is minimised. However, data along the crack face and tip are considered, thereby making a linear contour function significantly more susceptible to alignment errors; which is less of the case for the contour function as only gradients in the $x_{3}$ are considered.

Considering the noisy nature of DVC displacement data, reconstruction errors, crack smearing and crack segmentation difficulties, it is recommended that a contour function is chosen with a low gradient, i.e. with a large contour width. A 1 to $2 \%$ displacement error and a 1 to $2 \%$ crack tip location uncertainty equate to uncertainty in the computation of $J$ between 0.8 to 3.2 $\%$ for a crack opening mode. This error significantly increases for shear type loading to approximately 5 to $18.5 \%$.

\section{Verification on experimentally obtained DVC displacement fields}

The previous section highlighted two prominent sources of error that are related explicitly to DVC computed displacement data. It was shown that in the volumetric form, $J$ is sensitive to mixed-mode data, for which displacement noise and crack alignment can play an important 
role. Careful consideration in $q$ allows for less sensitivity concerning crack tip alignment in the crack propagation $\left(\boldsymbol{x}_{1}\right)$ and crack plane $\left(\boldsymbol{x}_{2}\right)$ directions. To test the robustness of the presented method, a material with natural contrast pattern was studied under mixed-mode loading. Magnesium alloy (WE43), which has high commercial value due to its good castability, high strength, high creep resistance, high corrosion resistance and high flame (ignition) resistance, was chosen. WE43 is widely used for structural components in aircraft for weight savings, and orthopaedic implants due to its biocompatibility [33].

\subsection{Materials}

Magnesium alloys, such as WE43 that contains $4 \mathrm{wt} \% \mathrm{Yt}$ and $3 \mathrm{wt} \% \mathrm{Nd}$, produce good speckle contrast in X-CT reconstructions due to the attenuation variations between the Magnesium bulk material and precipitates that are richer in rare earth elements [34,35] and is therefore well suited for DVC [36]. Precipitates with strong X-ray attenuation are quite visible in the reconstructed image (shown by a central slice in Fig. 7b). Brightening effects close to the edges of the sample and the notch should not be mistaken for variations in precipitate density. Rather, this is due to beam-hardening, which is a common (and persistent in this case) X-CT image artefact. The beam hardening is a constant artefact in this dataset, and so did had little effect the DVC analysis [37].

The specimen geometry is shown in Fig. 7. The specimen was rectangular over the gauge region of $50 \mathrm{~mm}$ in length $\left(\boldsymbol{X}_{3}\right)$. The gauge cross-section had a width $\left(\boldsymbol{X}_{1}\right)$ and thickness $\left(\boldsymbol{X}_{2}\right)$ of $10 \times 20 \mathrm{~mm}$ respectively. The notch was machined using wire-Electrode Discharge Machining (wire-EDM with a $0.3 \mathrm{~mm}$ diameter wire) to minimize residual stresses and work hardening that would result from mechanical notch cutting methods. The notch was machined at an angle of $30^{\circ}$ to each of the global axes $\boldsymbol{X}_{i}$. The loading of the sample was such that the bottom end was loaded in shear in the $\boldsymbol{X}_{1}$ direction under displacement control using a translation stage with an accuracy of $0.01 \mathrm{~mm}$.

\subsection{Tomography}

Tomographs were obtained using Stellenbosch University's X-CT scanner facility using a General Electric Phoenix VTomeX L240 microCT scanner with a $2048 \times 2048$ pixel detector with a 16-bit depth [38]. Optimized parameters were selected according to the guidelines set out in [39]. X-ray settings included $220 \mathrm{kV}$ and $200 \mu \mathrm{A}$ and copper beam filtration of $1.5 \mathrm{~mm}$, with a voxel size set to $15 \mu \mathrm{m}$. Image acquisition time was $500 \mathrm{~ms}$ per image and images were recorded in 2000 rotation steps during a full 360-degree rotation of the sample. At each step, the first image was discarded, and the subsequent three images averaged to provide high image quality. Detector shift was activated to minimize ring artefacts, and automatic scan optimizer was activated to eliminate artefacts due to possible sample movement or X-ray spot drift. Reconstruction was performed in system-supplied Datos reconstruction software.

Three X-CT images were taken to obtain two DVC datasets: one loaded reference image, one unloaded image with an applied displacement of $1 \mathrm{~mm}$ in $\boldsymbol{X}_{1}$ at the bottom grip region while the top grip remainedfixed, and one unloaded image with a rigid body displacement of $1 \mathrm{~mm}$. All displacements were assumed to be elastic as correlation was taken with reference to the loaded image. 
A centralized region consisting of $1260 \times 1860 \times 2024$ voxels $\left(\boldsymbol{X}_{1}, \boldsymbol{X}_{2}\right.$ and $\left.\boldsymbol{X}_{3}\right)$ was extracted and reduced to 8 bits for improved computational efficiency when computing DVC displacements (Fig. 7a). The crack geometry was inferred from the known notch geometry and the tomography images. To improve its estimate, a best-fitting linear plane was fitted by using a least-squares approach, whereby the crack front shape was assumed linear between $P_{i}^{1}$ and $P_{i}^{2}$ with constant $N_{i}$ and $M_{i}$ vectors.

\subsection{DVC}

The DVC analysis was performed with DaVis (ver. 8.3) volume correlation software using a fast Fourier transform (FFT). Image correlation used an initial subset size of 200 and a final subset size of 80 voxels and a step size of 20 voxels (75\% overlap). The rigid body displacement dataset revealed an experimental accuracy of $1.4 \mu \mathrm{m}$ (i.e. $\sim 0.1$ voxels), which is comparable to precision reported in the literature on a similar subset size [21,40]. Background noise was calculated as $0.36 \mu \mathrm{m}$ by correlating the two identically loaded datasets. The $U_{1}$ displacement field over the ROI is shown in Fig. 8a at $X_{1}=0$ and $X_{2}=0$.

The DVC datasets resulted in $44 \times 74$ × 60 displacement data points $\left(\boldsymbol{X}_{1}, \boldsymbol{X}_{2}\right.$ and $\left.\boldsymbol{X}_{3}\right)$. The computed DVC displacement data and crack geometry were imported into the Matlab environment, following rigid body rotation removal using the Euler angle extraction methodology of Mostafavi et al. [36].

\subsection{Finite element simulation}

Three-dimensional finite element (FE) simulation of the experimental setup was carried out using Abaqus ver. 6.13. The elastic properties of Mg WE43 were taken as provided by the manufacturer with Young's modulus of $44 \mathrm{GPa}$ and a Poisson's ratio of 0.35 . The linear-elastic model comprised of 105200 quadratic 20-node brick elements (C3D20) as shown in Fig. 9. The model used quadratic elements with quarter-point elements along the crack front, an initial radial element width of $0.3 \mathrm{~mm}$ at the notch mouth, converging to $0.01 \mathrm{~mm}$ (after 10 rings of elements) at the crack front, as shown in the magnified window in Fig. 9. Displacement boundary conditions were applied using the displacements applied at the BC. This way, the specimen loading could be simulated as close as possible to the experimental setup. Subsequently, $J$ values were computed using Abaqus' built-in functionality over ten contours, termed as $J_{\text {Abaqus. }}$. In parallel, the FE computed nodal displacements and the crack front geometry were imported into the Matlab environment for processing using the procedure presented in Section 3.

\section{$5.5 \mathrm{~J}$-integral computation}

The crack segment between $P_{i}^{1}$ and $P_{i}^{2}$ and integration volume $V$ are shown in Fig. 8b. The integration volume thickness was chosen as $\Delta S=0.6 \mathrm{~mm}$ (i.e. approximately three DVC data points in $\boldsymbol{x}_{3}$ ), and width and height was $\Delta x=4.8 \mathrm{~mm}$ thus considering nine $J$ computation points along the crack path (i.e. in $\boldsymbol{x}_{1}$ and $\boldsymbol{x}_{2}$ ). It is estimated that the crack tip alignment concerning the integration volume was $<1 \%$ (to 5 voxels or $75 \mu \mathrm{m}$ ), as the notch tip of radius $150 \mu \mathrm{m}$ was easily visible in the Tomographs. A crack mask $\hat{r}_{m}=1.2 \mathrm{~mm}$ (i.e. four subsets) was used. $J$ values were computed using the contour function. 
Fig. 10 shows the DVC computed displacement data over the integration volume $\mathrm{V}$ at $\hat{x}_{3}=0$. The data shows the mixed-mode nature of the crack loading, whereby significant mode III type loading is present. This is to be expected as the sample loaded in shear perpendicular to the notch tip.

Both DVC and FE displacement data were used to compute $J$ along the defined crack (termed $J_{D V C}$ and $J_{F E}$ respectively) using the procedure as outlined in Section 3. Both DVC and FE displacement data was chosen to compare best: i) the course mesh of the presented approach with an ideally meshed notch tip (FE) and; ii) estimate the influence of experimental error (displacement noise and crack tip position).

\subsection{Results and discussion}

The mean normalised $J$ computations are shown in Fig. 11a for the equivalent DVC, FE, and Abaqus computations. Fig. $11 \mathrm{~b}$ illustrates the contour independence for the first four computations along the crack path for the DVC displacement data. The difference when computing $J$ from FE and DVC data is $6.2 \%$ indicating the combined error of displacement noise and crack tip position uncertainty. This is in agreement with the estimated error, presented in Section 4, of $7 \%$. The experimental error in the computation of $J$ is approximated correlating the loaded and two unloaded datasets (not shown) resulting in a maximum deviation of $5.2 \%$, indicating the strong influence of DVC displacement noise when computing $J$ from DVC displacement data.

Considering the differences between the FE and Abaqus $J$ computations a smoothing effect near the edges of the specimen is highlighted. The relatively large integration volume of 4.8 $\mathrm{mm}$, when considering that the Abaqus contours are taken $0.1 \mathrm{~mm}$ from the crack tip, results in less sensitivity in capturing variations in $J$ along the crack front. This is attributed to the larger integration volume being less sensitive to localised strain gradients, which are more apparent near the edge of the specimen where the stress-strain conditions change from plane strain to plane stress.

The agreement between the measured and predicted $J$ values demonstrates the effectiveness of using DVC data to obtain fracture parameters of the loaded sample. This study shows that highresolution X-CT can be used to reliably quantify the energy release rate, measured employing the J-integral. The study furthermore highlights that $J$ is highly effective for the computations of fracture properties in the volume when using DVC data. Whiel sensitive to displacement noise, $J$ does not require the exact knowledge of the crack tip position. When a mode I type loading is expected, an error less than $1 \%$ can be expected in $J$. This is especially advantageous for materials where the location of the crack tip is difficult to identify, such as nuclear graphite [19].

\section{Conclusions}

A simple procedure for evaluating the J-integral in its volumetric form from DVC computed displacement data has been presented and verified against experimental data for a mixed-mode loaded crack. DVC computed displacement data present unique challenges due to noise, erroneous data near crack faces and imprecise knowledge of the crack tip position. 
- The procedure for the robust extraction of $J$-integral values uses a specifically defined $q$ function, along a three-dimension crack front.

- Common errors associated with DVC computed displacement data were quantified using analytical displacement data to assign confidence intervals. In the presented form, the J-integral shows sensitivity to displacement noise; however, it does not require the exact knowledge of the crack tip.

- Analysis of the J-integral obtained from an experimental study of a mixed-mode problem, using a Magnesium WE43 alloy with an inclined single edge rounded notch to represent a crack, found a good agreement with the analytical solution from an FE model. J-integral values along the crack front could be determined to within $6.2 \%$ of the finite element solution.

\section{Acknowledgments}

The authors disclosed receipt of the following financial support for the research, authorship, and publication of this article: This work is based on the research supported in part by the National Research Foundation of South Africa for the grant, Unique Grant No. 87955 and 106932, and in part by the ESKOM Power Plant Engineering Institute (EPPEI) Material Science Specialisation programme.

\section{References}

1. McNeill, S.R., Peters, W.H., and Sutton, M. a. (1987) Estimation of stress intensity factor by digital image correlation. Eng. Fract. Mech., 28 (1), 101-112.

2. Lim, I.L., Johnston, I.W., and Choi, S.K. (1992) Comparison between various displacement-based stress intensity factor computation techniques. Int. J. Fract., 58 (3), 193-210.

3. Roux, S., and Hild, F. (2006) Stress intensity factor measurements from digital image correlation: post-processing and integrated approaches. Int. J. Fract., 140 (1-4), 141157.

4. Rethore, J., and Gravouil, A. (2005) Estimation of mixed-mode stress intensity factors using digital image correlation and an interaction integral. Int. J. ..., 65-79.

5. Yoneyama, S., Morimoto, Y., and Takashi, M. (2006) Automatic evaluation of mixedmode stress intensity factors utilizing digital image correlation. Strain, 42 (1), 21-29.

6. Yoneyama, S., Ogawa, T., and Kobayashi, Y. (2007) Evaluating mixed-mode stress intensity factors from full-field displacement fields obtained by optical methods. Eng. Fract. Mech., 74 (9), 1399-1412.

7. Sistaninia, M., Ayatollahi, M.R., and Sistaninia, M. (2014) On fracture analysis of cracked graphite components under mixed mode loading.

8. Yoneyama, S., Arikawa, S., Kusayanagi, S., and Hazumi, K. (2014) Evaluating Jintegral from displacement fields measured by digital image correlation. Strain, 50 (2), 147-160.

9. Molteno, M.R., and Becker, T.H. (2015) Mode I - III Decomposition of the J -integral from DIC Displacement Data. Strain, 51 (6), 492-503.

10. Becker, T.H., Mostafavi, M., Tait, R.B., and Marrow, T.J. (2012) An approach to calculate the $\mathrm{J}$-integral by digital image correlation displacement field measurement. Fatigue Fract. Eng. Mater. Struct., 35 (10), 971-984.

11. Poissant, J., and Barthelat, F. (2010) A novel "subset splitting" procedure for digital 
image correlation on discontinuous displacement fields. Exp. Mech., 353-364.

12. Barhli, S.M., Mostafavi, M., Cinar, A.F., Hollis, D., and Marrow, T.J. (2017) J-Integral calculation by finite element processing of measured full-field surface displacements. Exp. Mech., 1-13.

13. Yoneyama, S. (2011) Smoothing measured displacements and computing strains utilising finite element method. Strain, 47 (SUPPL. 2), 258-266.

14. Mathieu, F., Hild, F., and Roux, S. (2013) Image-based identification procedure of a crack propagation law. Eng. Fract. Mech., 103, 48-59.

15. Cinar, A.F., Barhli, S.M., Hollis, D., Flansbjer, M., Tomlinson, R.A., Marrow, T.J., and Mostafavi, M. (2017) An autonomous surface discontinuity detection and quantification method by digital image correlation and phase congruency. Opt. Lasers Eng., 96 (May), 94-106.

16. Amiot, F., Bornert, M., Doumalin, P., Dupré, J.-C., Fazzini, M., Orteu, J.-J., Poilâne, C., Robert, L., Rotinat, R., Toussaint, E., Wattrisse, B., and Wienin, J.S. (2013) Assessment of Digital Image Correlation Measurement Accuracy in the Ultimate Error Regime: Main Results of a Collaborative Benchmark. Strain, 49 (6), 483-496.

17. Maire, E., and Withers, P.J. (2014) Quantitative X-ray tomography. Int. Mater. Rev., 59 (1), 1-43.

18. Bay, B.K. (2008) Methods and applications of digital volume correlation. J. Strain Anal. Eng. Des., 43 (8), 745-760.

19. Becker, T.H., Marrow, T.T.J., Tait, R.R.B., and Mostafavi, M. (2011) Damage, crack growth and fracture characteristics of nuclear grade graphite using the Double Torsion technique. J. Nucl. Mater., 414 (1), 32-43.

20. Molteno, M.R., and Becker, T.H. (2015) Mode I-III Decomposition of the J -integral from Digital Image Correlation Displacement Data. Int. Conf. Comput. Model. Fract. Fail., 1-2.

21. Mostafavi, M., McDonald, S. a., Mummery, P.M., and Marrow, T.J. (2013) Observation and quantification of three-dimensional crack propagation in poly-granular graphite. Eng. Fract. Mech., 44, 1-11.

22. Shih, C.F., Moran, B., and Nakamura, T. (1986) Energy release rate along a threedimensional crack front in a thermally stressed body. Int. J. Fract., 30, 79-102.

23. Dassault Systèmes Simulia, Fallis, A.., and Techniques, D. (2013) ABAQUS documentation. Abaqus 6.12, 53 (9), 1689-1699.

24. D'Errico, J. (2012) Distance based interpolation along a general curve in space. Mathworks file Exch.

25. Rannou, J., Limodin, N., Réthoré, J., Gravouil, A., Ludwig, W., Baïetto-Dubourg, M.C., Buffière, J.Y., Combescure, A., Hild, F., and Roux, S. (2010) Three dimensional experimental and numerical multiscale analysis of a fatigue crack. Comput. Methods Appl. Mech. Eng., 199 (21-22), 1307-1325.

26. Kuna, M. (2013) Numerical Calculation of Generalized Energy Balance Integrals, in Finite Elements in Fracture Mechanics, Springer Netherlands, pp. 263-304.

27. Amidror, I. (2002) Scattered data interpolation methods for electronic imaging systems: a survey. J. Electron. Imaging, 11 (2), 157.

28. Fornberg, B., and Zuev, J. (2007) The Runge phenomenon and spatially variable shape parameters in RBF interpolation. Comput. Math. with Appl., 54 (3), 379-398.

29. Garcia, D. (2010) Robust smoothing of gridded data in one and higher dimensions with missing values. Comput. Stat. Data Anal., 54 (4), 1167-1178.

30. Wang, G., Garcia, D., Liu, Y., de Jeu, R., and Johannes Dolman, A. (2012) A threedimensional gap filling method for large geophysical datasets: Application to global satellite soil moisture observations. Environ. Model. Softw., 30, 139-142. 
31. Münch, B., Trtik, P., Marone, F., and Stampanoni, M. (2009) Stripe and ring artifact removal with combined wavelet-Fourier filtering. EMPA Act., 17 (2009-2010 EMPA ACTIVITIES), 34-35.

32. Schreier, H., Orteu, J.J., and Sutton, M.A. (2009) Image correlation for shape, motion and deformation measurements: Basic concepts, theory and applications.

33. Klocke, F., Schwade, M., Klink, A., and Kopp, A. (2011) EDM machining capabilities of magnesium (Mg) alloy WE43 for medical applications. Procedia Eng., 19, 190-195.

34. Marrow, T.J., Mostafavi, M., Hashimoto, T., and Thompson, G.E. (2014) A quantitative three-dimensional in situ study of a short fatigue crack in a magnesium alloy. Int. J. Fatigue, 66, 183-193.

35. King, A., Ludwig, W., Herbig, M., Buffire, J.Y., Khan, A.A., Stevens, N., and Marrow, T.J. (2011) Three-dimensional in situ observations of short fatigue crack growth in magnesium. Acta Mater., 59 (17), 6761-6771.

36. Mostafavi, M., Collins, D.M., Cai, B., Bradley, R., Atwood, R.C., Reinhard, C., Jiang, X., Galano, M., Lee, P.D., and Marrow, T.J. (2015) Yield behavior beneath hardness indentations in ductile metals, measured by three-dimensional computed X-ray tomography and digital volume correlation. Acta Mater., 82, 468-482.

37. Mostafavi, M., Collins, D.M., Cai, B., Bradley, R., Atwood, R.C., Reinhard, C., Jiang, X., Galano, M., Lee, P.D., and Marrow, T.J. (2015) Yield behavior beneath hardness indentations in ductile metals, measured by three-dimensional computed X-ray tomography and digital volume correlation. Acta Mater., 82, 468-482.

38. du Plessis, A., le Roux, S.G., and Guelpa, A. (2016) The CT Scanner Facility at Stellenbosch University: An open access X-ray computed tomography laboratory. Nucl. Instruments Methods Phys. Res. Sect. B Beam Interact. with Mater. Atoms, 384, 42-49.

39. du Plessis, A., Broeckhoven, C., Guelpa, A., and le Roux, S.G. (2017) Laboratory Xray micro- computed tomography: a user guideline for biological samples. Gigascience, 6, 1-11.

40. Mostafavi, M., Baimpas, N., Tarleton, E., Atwood, R.C., McDonald, S.A., Korsunsky, A.M., and Marrow, T.J. (2013) Three-dimensional crack observation, quantification and simulation in a quasi-brittle material. Acta Mater., 61 (16), 6276-6289. 


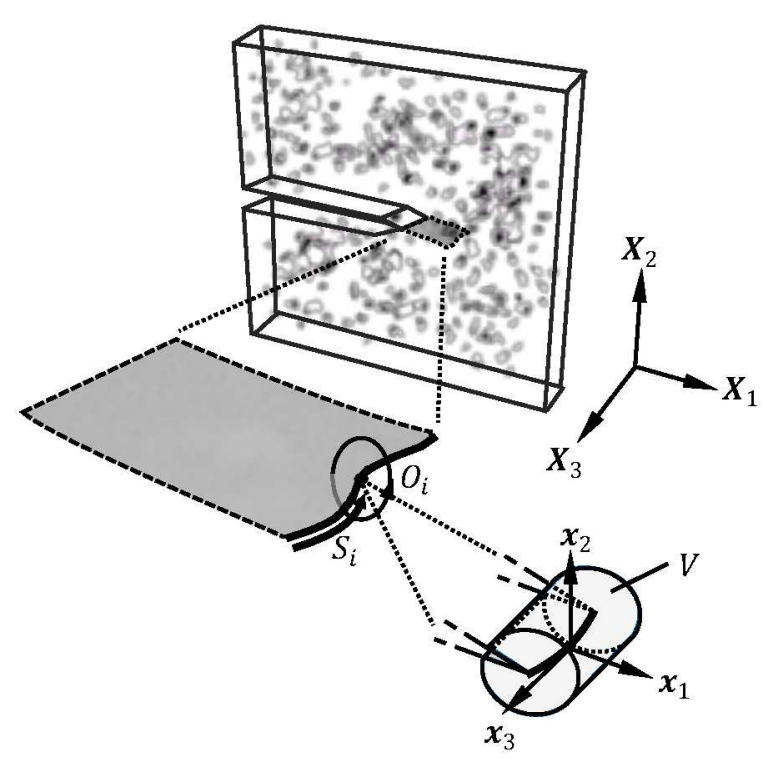

Fig 1. Cracked specimen, showing crack front $S_{i}$ and integration volume $V$ centred at $O_{i}$. 


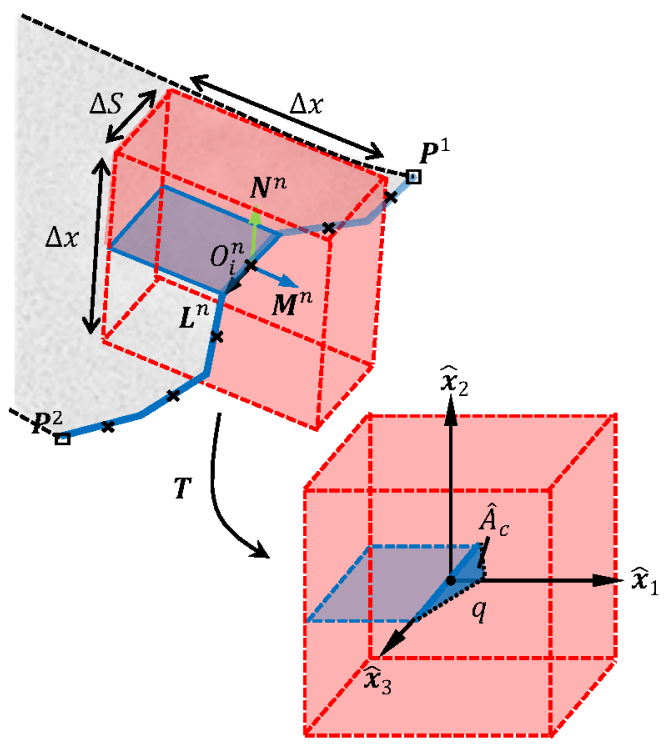

(a)

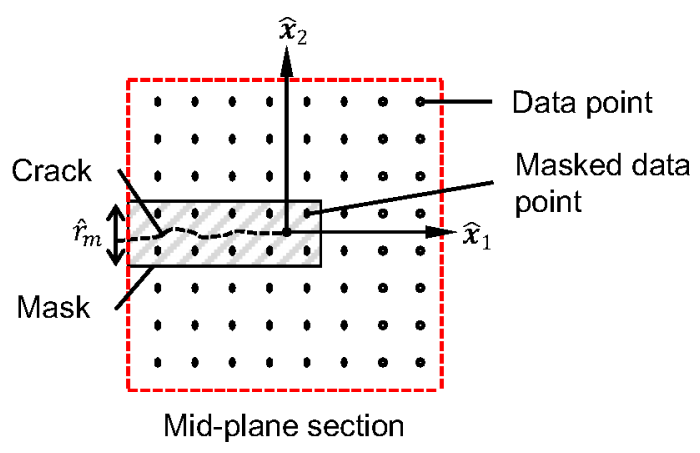

(b)

Fig. 1. (a) Crack front showing integration volume $V$ positioned at $O_{i}$ along $S_{i}$ transformed to the local coordinate position $\widehat{\boldsymbol{x}}_{i}$. (b) cross-section of the mid-plane of $V$ showing the regularised data points and crack mask of width $\hat{r}_{m}$. 

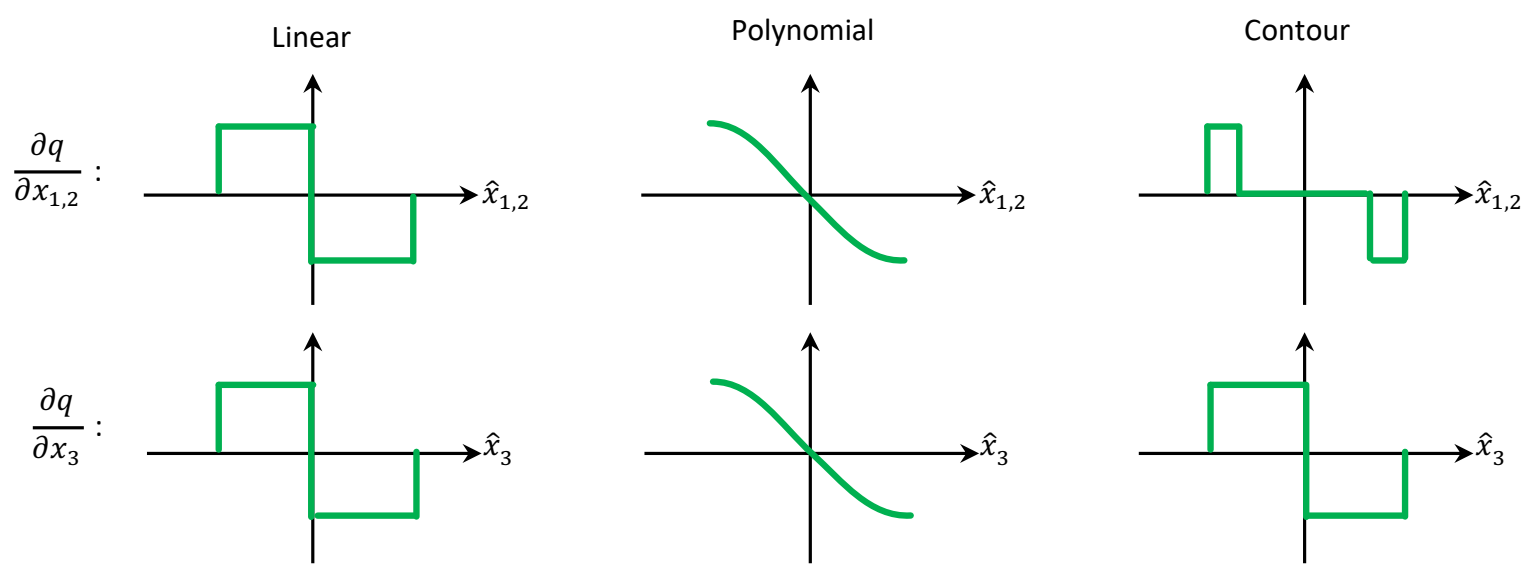
$\hat{A}_{c}:$
$\Delta S$
$\int 1-\widehat{x}_{3}^{4} d \widehat{x}_{3}$
$\Delta S$

Fig. 1. $q$ function gradients for linear, Polynomial (4th order) and contour (pricewise linear) functions. 


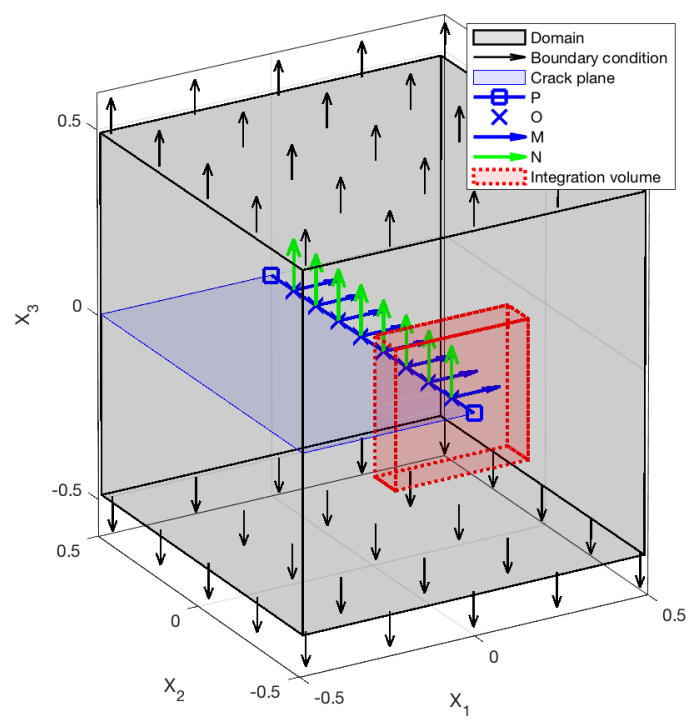

(a)

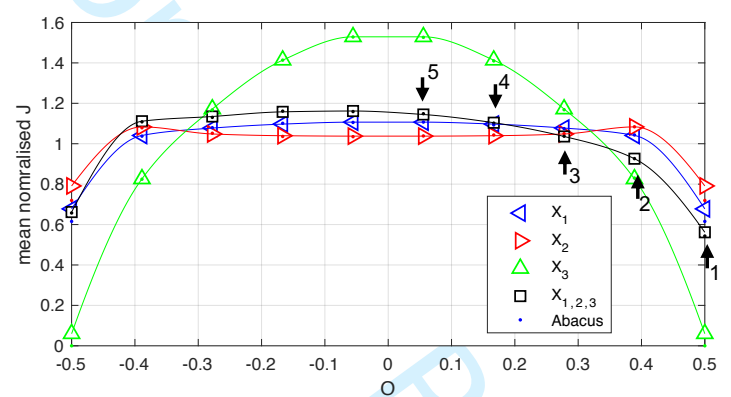

(b)

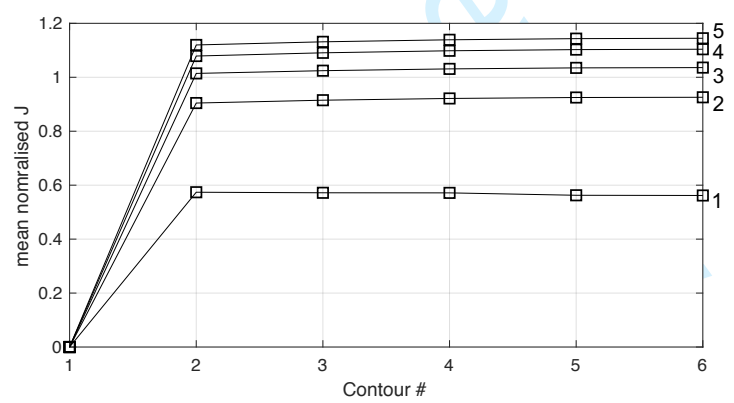

(c)

Fig. 4. (a) Unit volume showing boundary conditions (arrow), crack path P, crack segmentation and integration volume $V$. (b) showing the respective $J$ values commuted along the crack path $P$. (c) J contours for points $1-5$ shown in (b) indicating $J$ convergence. 

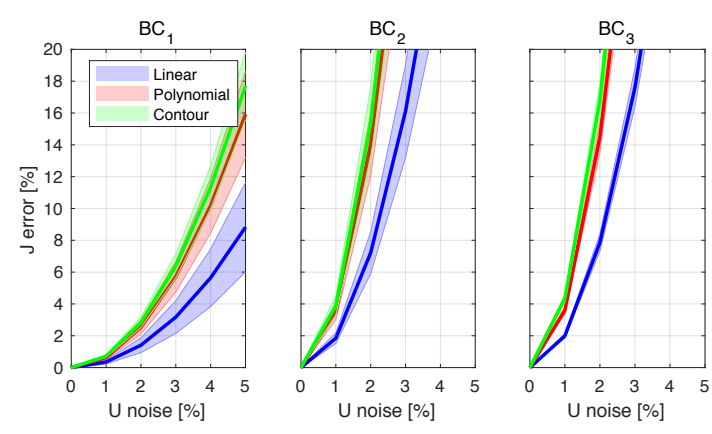

\begin{tabular}{|c|c|c|c|}
\hline & J error [\%] & J error [\%] & J error [\%] \\
\hline $1 \%$ & $0.72 \pm 0.079$ & $3.9 \pm 0.59$ & $4.4 \pm 0.24$ \\
\hline $2 \%$ & $2.9 \pm 0.32$ & $15.3 \pm 2.3$ & $16.8 \pm 1.1$ \\
\hline $5 \%$ & $18 \pm 2.1$ & $95 \pm 14$ & $103 \pm 6.9$ \\
\hline
\end{tabular}

Fig. 5. Influence of random displacement noise on the computation of J. The table below provides values for the contour q function. 

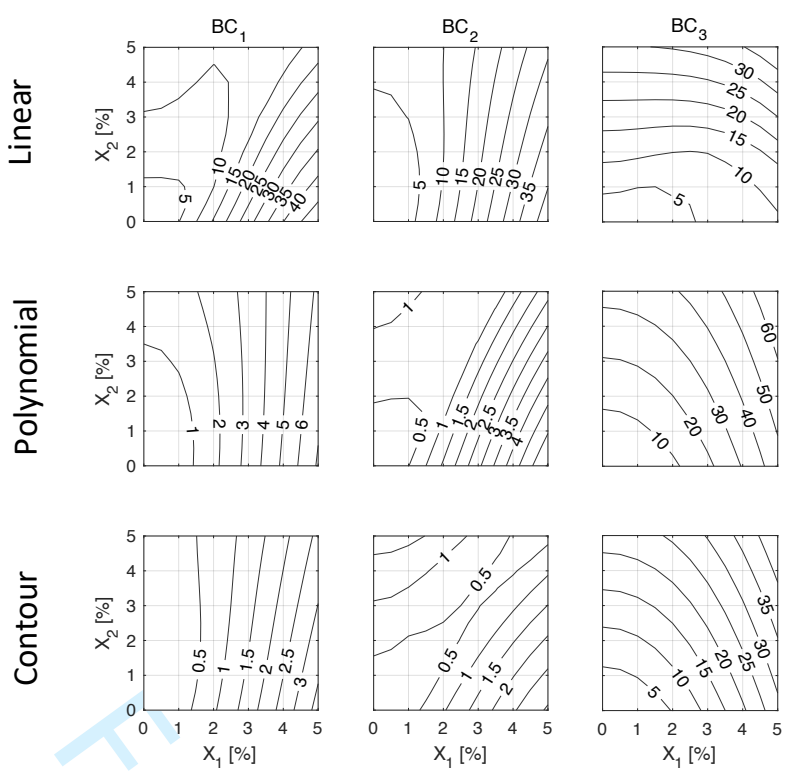

\begin{tabular}{|c|c|c|c|}
\hline & J error [\%] & J error [\%] & J error [\%] \\
\hline $1 \%$ & $0.20-0.10$ & $0.27-0.10$ & $5.2-1.3$ \\
\hline $2 \%$ & $0.72-0.27$ & $0.42-0.25$ & $13-3.3$ \\
\hline $5 \%$ & $2.6-0.84$ & $0.38-0.61$ & $44-9.8$ \\
\hline
\end{tabular}

Fig. 6. Influence of integration volume position relative to crack front location. The table below provides values for the contour q function. 


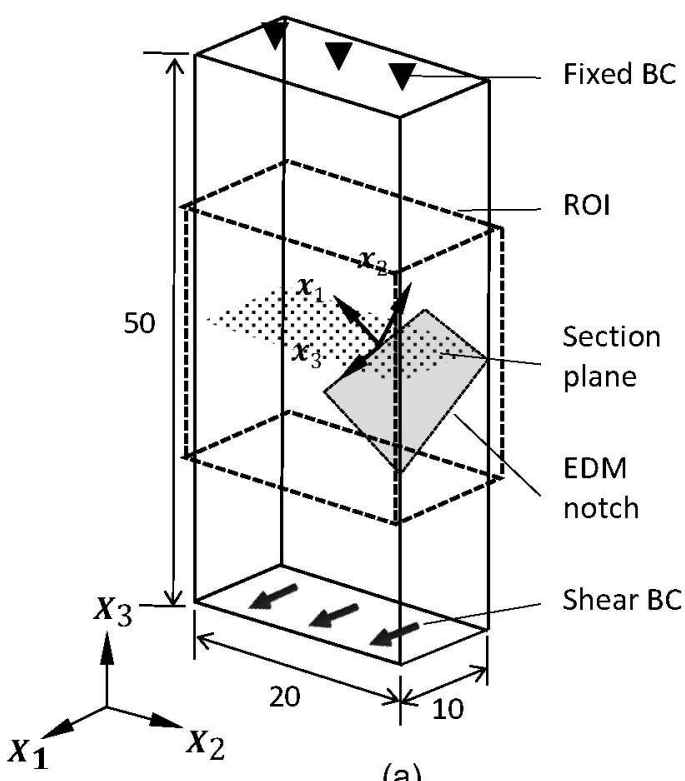

(a)
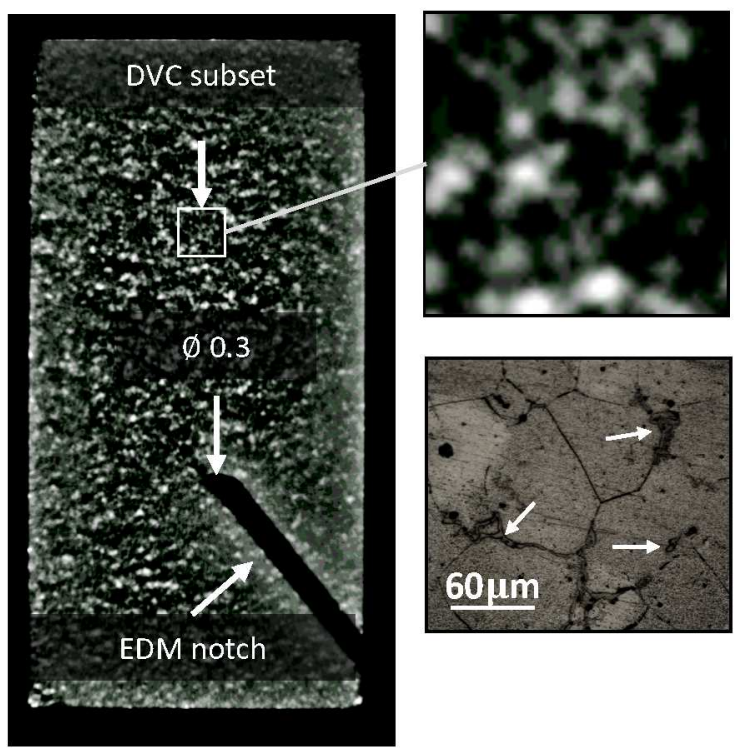

(b)

Fig. 7.(a) Schematic of mixed-mode specimen, highlighting BCs, ROI over which displacements were computed using DVC, and the mid-plane section. (b) X-CT scan image of mid-plane showing the EDM notch of $0.3 \mathrm{~mm}, \mathrm{DVC}$ subset and micrograph (highlighting contrasting features). 


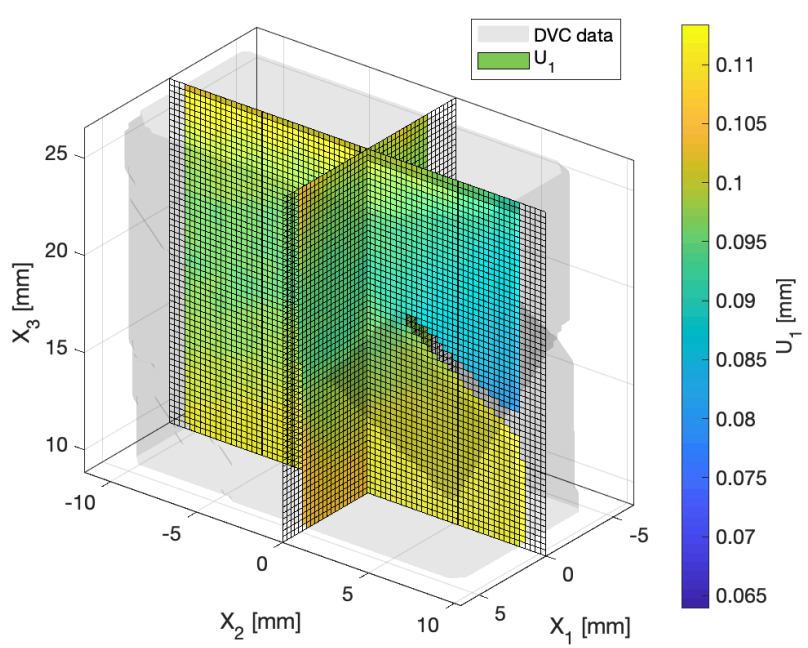

(a)

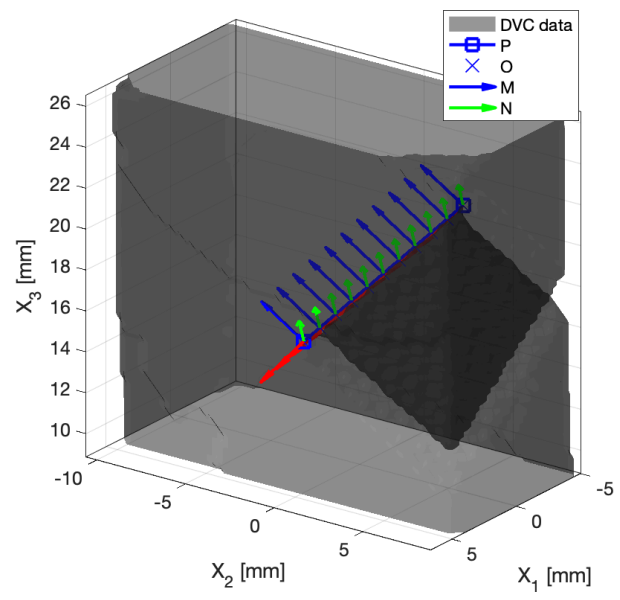

(b)

Fig. 8.(a) $U_{1}$ DVC computed displacement planes. (b) Analysis region showing the crack path $P$, crack segmentation centres $O_{i}$, and crack orientation defined by $M_{i}$ and $N_{i}$. 


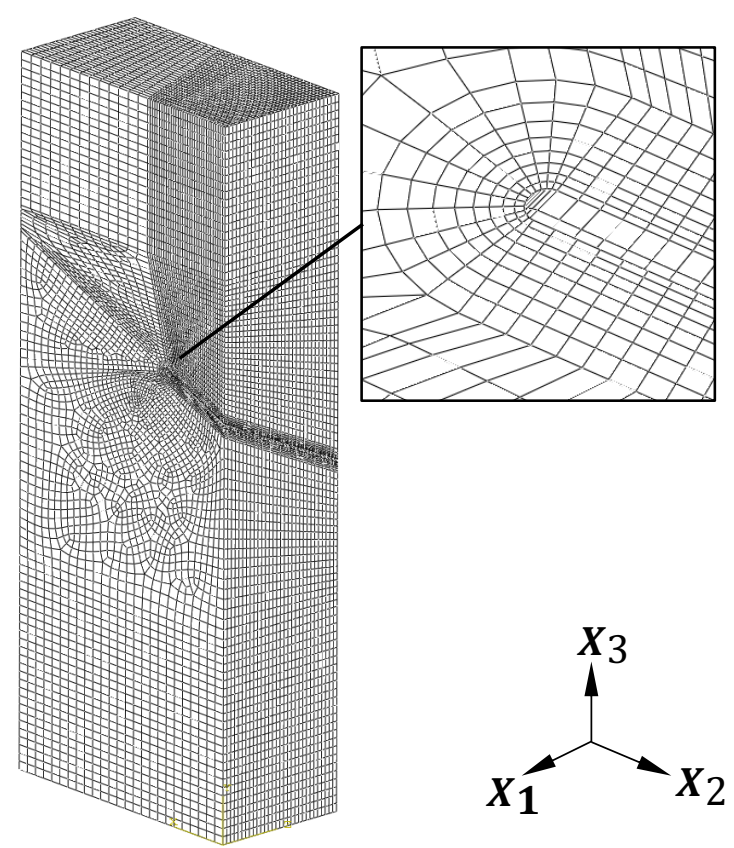

Fig. 9. Equivalent FE model of experimental setup. Insert showing notch tip mesh detail allowing for $10 \mathrm{~J}$ contours. 


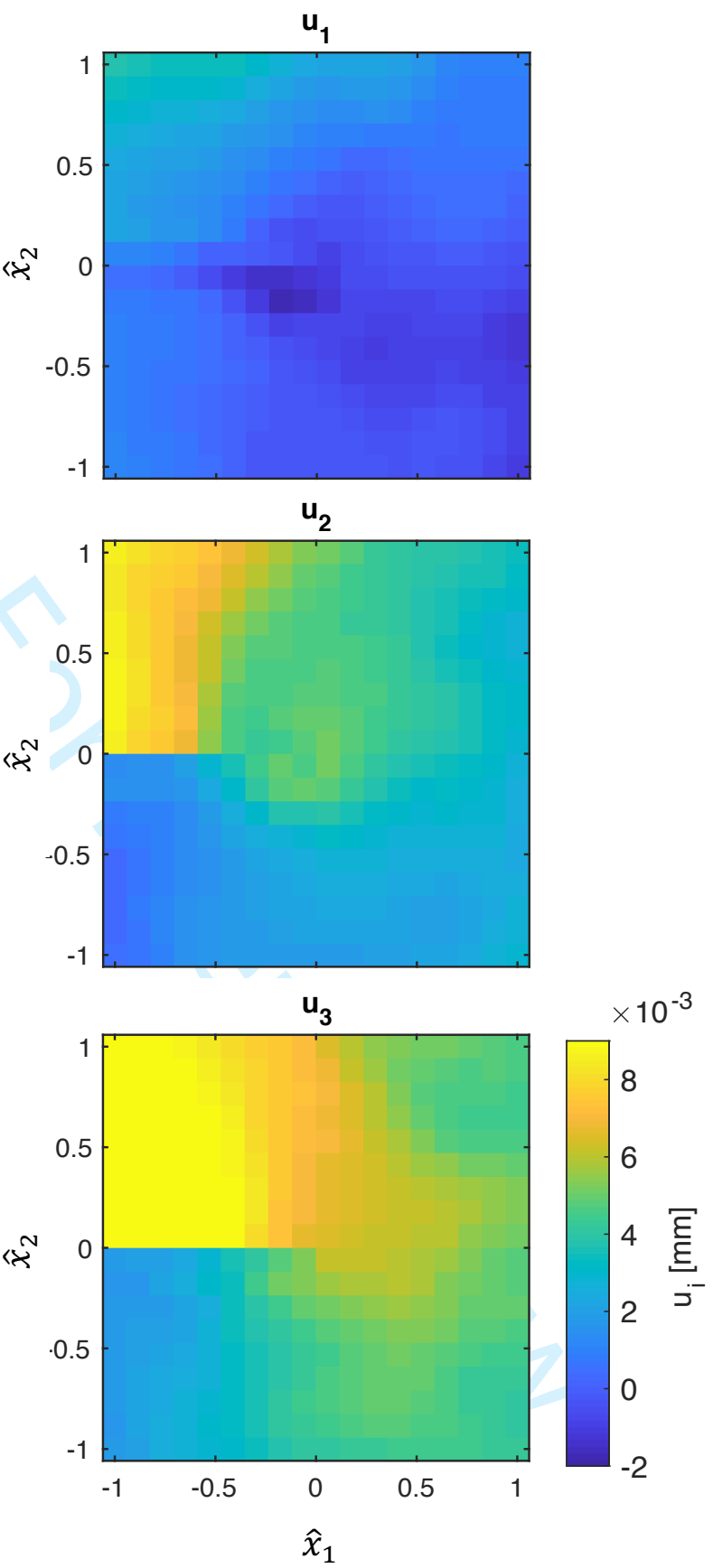

Fig. 10. DVC displacement for an integration volume at position $n=4$ (indicated in Fig. 11). Displacements are shown for at $x_{3}=0$. 


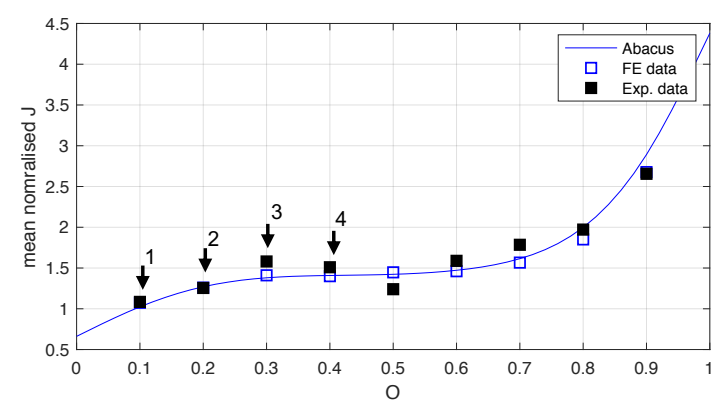

(a)

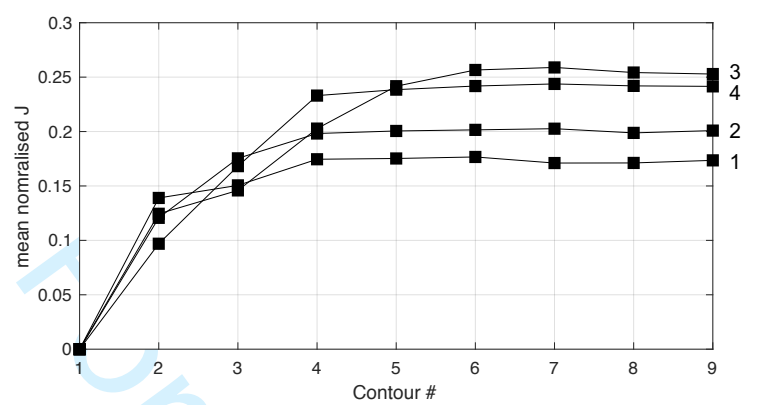

(b)

Fig. 11. Mean normalised $J$ values along the crack path from the experiment and equivalent FE model. (b) J contours for points $1-4$ shown in (a) indicating $J$ convergence. 